\title{
Post common envelope binaries from the SDSS
}

\section{SDSS J120615.73+510047.0: a new low accretion rate magnetic binary}

\author{
A. D. Schwope ${ }^{1}$, A. Nebot Gomez-Moran ${ }^{1}$, M. R. Schreiber ${ }^{2}$, and B. T. Gänsicke ${ }^{3}$ \\ 1 Astrophysikalisches Institut Potsdam, An der Sternwarte 16, 14482 Potsdam, Germany \\ e-mail: aschwope@aip.de \\ 2 Departamento de Física y Astronomía, Universidad de Valparaíso, Avenida Gran Bretana 1111, Valparaíso, Chile \\ ${ }^{3}$ Department of Physics, University of Warwick, Coventry CV4 7AL, UK
}

Received 22 January 2009 / Accepted 18 March 2009

\section{ABSTRACT}

\begin{abstract}
We report the discovery of the ninth pre-polar consisting of a late-type ZAMS secondary and a magnetic white dwarf. The white dwarf accretes at an extreme low rate, $\dot{M} \sim 10^{-14} M_{\odot} \mathrm{yr}^{-1}$, from the wind of the companion donor star. The source was found in our systematic search for WD/MS binaries within SDSS/SEGUE. Based on seven Sloan spectra we estimate a binary period of 200, 230, or $270 \mathrm{~min}$. The UV to IR spectral energy distribution was decomposed into a dM3-dM4 ZAMS secondary and a cool white dwarf, $\sim 9000 \mathrm{~K}$, which consistently imply a distance between 360 and $420 \mathrm{pc}$. The optical spectrum displays one pronounced cyclotron hump, likely originating from a low-temperature plasma, $\sim 1 \mathrm{keV}$, in a field of $108 \mathrm{MG}$. We comment on the evolutionary link between polars and pre-polars.
\end{abstract}

Key words. accretion, accretion disks - stars: magnetic fields - binaries: close - stars: individual: SDSS J120615.73+510047.0

\section{Introduction}

Reimers et al. (1999) discovered a white-dwarf/main sequence binary with a very peculiar emission line, while inspecting spectra of quasar candidates in the HQS objective prism survey. The line turned out to be the third harmonic of a cyclotron fundamental emitted by a low-density plasma in a system that was regarded a magnetic cataclysmic variable (AM Herculis star or a polar) in a persistent low state. Shortly thereafter, Reimers \& Hagen (2000) found a second system with very similar properties, which led Schwope et al. (2002b) to call them LARPs, Low Accretion Rate Polars.

In the ensuing years six further objects of this kind were uncovered in the SDSS (Schmidt et al. 2005, 2007) that share the following properties. They host active late-type main-sequence stars and accreting, cool, magnetic white dwarfs. All display pronounced cyclotron spectra originating from low-density plasmas on the white dwarfs. The accretion rates are of the order of $10^{-13} M_{\odot} \mathrm{yr}^{-1}$, orders of magnitude below the rates expected for polars at the given orbital periods, which are about $10^{-10} M_{\odot} \mathrm{yr}^{-1}$. The low accretion rates appeared to be constant over years (Schwarz et al. 2001) and were found to be consistent with the wind mass loss rate of the active secondary (Schwope et al. 2002b).

The low accretion rates together with the system parameters of some well-studied systems suggested a scenario of underfilling secondaries and no Roche-lobe accretion at all. Hence, their class name, referring to them as polars, seems to be a misnomer.

The class is still very small, most of their members were serendipituously found as quasar candidates due to their unusual colors or their broad (cyclotron) emission lines. Here we report the first detection of such an object in a project targeting white-dwarf/main-sequence (WDMS) binaries spectroscopically following a multi-colour photometric selection process within SDSS/SEGUE (Schreiber et al. 2007) (see also Rebassa-Mansergas et al. 2007; Nebot Gómez-Morán et al. 2009; Schreiber et al. 2009; Yanny et al. 2009, for a more comprehensive description of the survey).

Within SDSS/SEGUE (for a technical description of the SDSS survey see Fukugita et al. 1996; Gunn et al. 1998, 2006; Hogg et al. 2001; Ivezić et al. 2004; Pier et al. 2003; Smith et al. 2002; Stoughton et al. 2002; Tucker et al. 2006; York et al. 2000) we obtained 533 spectra from a multi-color selected sample designed to find WDMS binaries with cool white dwarfs and late-type secondaries. While routinely attempting a spectral decomposition into its stellar constituents, SDSS J120615.73+510047.0 attracted more interest due to a prominent spectral hump on top of the blue continuum of a suspected white dwarf. We thus retrieved not only the mean SDSS/SEGUE spectrum but also the seven individual spectra from the SDSS data base. As a result of this more comprehensive analysis we are confident that SDSS J120615.73+510047.0 is the ninth member of the elusive class of WDMS binaries with a magnetic white dwarf.

We present the system parameters as derived from the Sloan archive and discuss the class properties.

\section{SDSS observations and analysis}

With ugriz magnitudes of 20.58, 19.90, 19.32, 18.14, 17.39, SDSS J120615.73+510047.0 was just slightly brighter than our chosen limit for SDSS-spectroscopy, $g=20$ (Schreiber et al. 2007). 


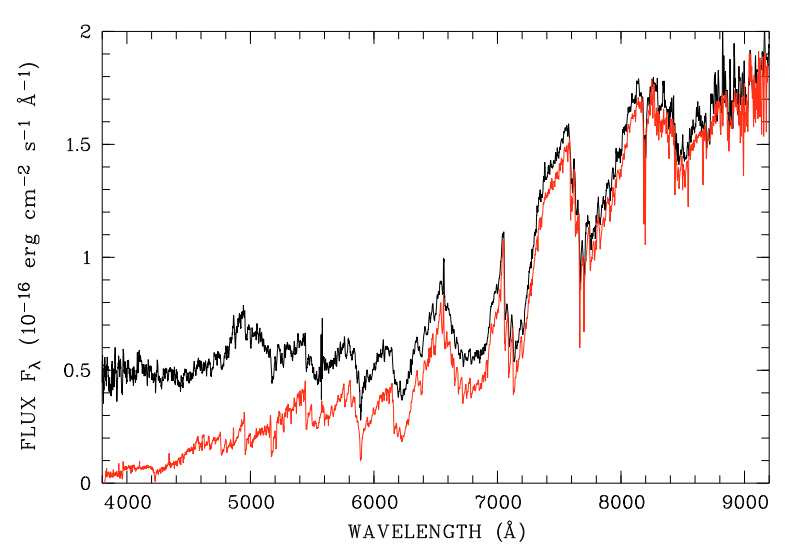

Fig. 1. Mean SDSS spectrum of SDSS J120615.73+510047.0. The spectrum was boxcar filtered over three pixels. The spectrum shown in red is a dM4-template spectrum constructed from SDSS data.

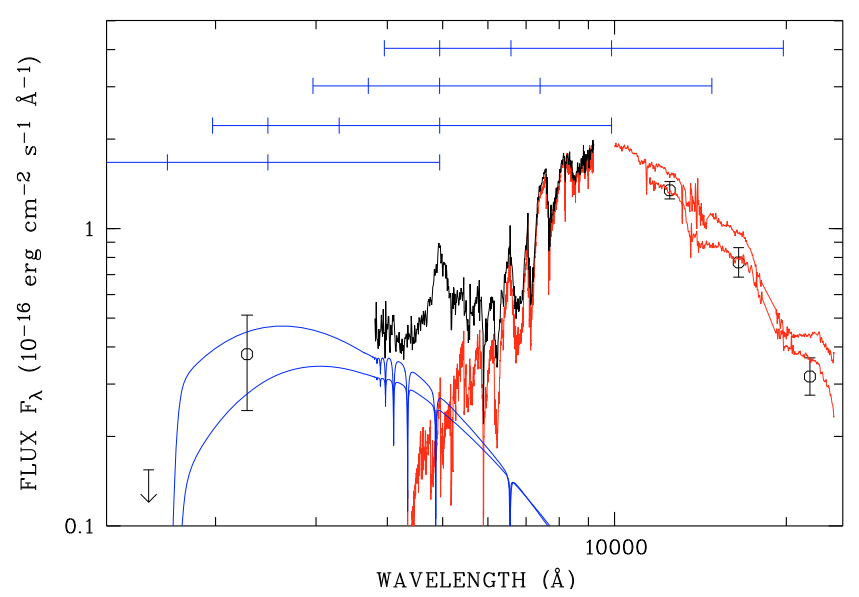

Fig. 2. Spectral energy distribution of SDSS J120615.73+510047.0. In black are shown data obtained from 2MASS (JHK photometry), SDSS (optical spectrum), and GALEX (UV photometry). Spectral templates for the secondary obtained from the SDSS (optical) and from Leggett et al. (2000) are shown in red. DA model spectra for $8000 \mathrm{~K}$ and $9000 \mathrm{~K}$ are shown in blue. The expected locations of the first five harmonics in fields of 216, 108, 72, and $54 \mathrm{MG}$ are indicated by short ticks in the upper part of the diagram.

\subsection{Average SDSS spectrum and the spectral energy distribution}

The average spectrum of SDSS J120615.73+510047.0 published in SDSS-DR7 (Abazajian et al. 2009) is shown in Fig. 1. It is dominated by the late-type secondary star whose spectral features are best reflected by a dM4 ZAMS template spectrum. The distance to SDSS J120615.73+510047.0 derived from a scaled dM4 template spectrum is $d_{\mathrm{MS}}=420 \pm 120 \mathrm{pc}$. SDSS J120615.73+510047.0 was detected also with 2MASS and the IR colours and the IR-to-optical spectral energy distribution suggest a slightly earlier spectral type, M3. Figure 2 shows the SDSS spectrum and also contains a suitably scaled M4 spectral template in the optical and IR spectra of LHS57 (M4) and LHS54 (M3), both taken from Legget's spectral archive (Leggett et al. 2000) scaled to the same optical brightness. While an M3type secondary and corresponding larger distance is consistent with the data, we nevertheless use an M4 template for the spectral deconvolution of the SDSS-spectra.
Table 1. Time of mid-integration of the seven sub-spectra obtained on March 9/10, 2008, phases according to Eq. (1) and radial velocity of the NaI doublet.

\begin{tabular}{rcrr}
\hline \hline $\begin{array}{r}\text { Seq. } \\
\#\end{array}$ & $\begin{array}{c}\text { Time } \\
(\text { HJD })\end{array}$ & Phase & $\begin{array}{r}\text { Velocity } \\
\left(\mathrm{km} \mathrm{s}^{-1}\right)\end{array}$ \\
\hline 1 & 2454536.246827 & 0.77 & -300 \\
2 & 2454536.328123 & 0.36 & 264 \\
3 & 2454537.220600 & 0.86 & -188 \\
4 & 2454537.239975 & 0.00 & 49 \\
5 & 2454537.259523 & 0.14 & 195 \\
6 & 2454537.284940 & 0.33 & 227 \\
7 & 2454537.299859 & 0.43 & 151 \\
\hline
\end{tabular}

The $1 \sigma$ measurement uncertainty is $9 \mathrm{~km} \mathrm{~s}^{-1}$.

The field of SDSS J120615.73+510047.0 was observed for 105 s with GALEX, and SDSS J120615.73+510047.0 was detected in the NUV channel but not detected in the FUV channel. The GALEX fluxes ( $1 \sigma$ upper limit for the FUV channel) are included in Fig. 2, too.

The residual SDSS-spectrum after subtraction of the M-star template can be described by a blue featureless continuum with a broad blue hump superimposed. The absence of any absorption line suggests a classification as DC white dwarf (but see the discussion below). Nevertheless, we use a DA model atmosphere as a first order approximation for the white-dwarf spectral flux. The optical to ultra-violet spectral energy distribution is consistent with a low-temperature white dwarf of 8000-9000 K (see Fig. 2). Assuming $9000 \mathrm{~K}$ and an average white dwarf of $M_{\mathrm{wd}}=0.6 M_{\odot}$ and $R_{\mathrm{wd}}=8.3 \times 10^{8} \mathrm{~cm}$ the distance to SDSS J120615.73+510047.0 is $d_{\mathrm{WD}}=360 \mathrm{pc}$, in good agreement with $d_{\text {MS }}$.

\subsection{Line variability}

The SDSS/SEGUE spectrum of SDSS J120615.73+510047.0 published via SDSS-DR7 is the average of seven sub-spectra with exposure of $15 \mathrm{~min}$ each obtained during the two nights of March 9 and 10, 2008 (see Table 1 for exact dates). We retrieved the individual spectra from the Sloan database to search for spectral and photometric variability.

$\mathrm{H} \alpha$ emission and $\mathrm{NaI}$ absorption lines were used to search for radial velocity variations by fitting Gaussians to the observed data. The double Gaussian for the NaI lines was fitted with fixed separation between the two lines and with the same width of both lines. The line fluxes of both $\mathrm{H} \alpha$ emission and $\mathrm{NaI}$ absorption lines show insignificant variability. Both line features are resolved with a measured $F W H M$ of $\sim 5 \AA(\mathrm{H} \alpha)$ and $6.5 \AA(\mathrm{NaI})$. Again, variability of the width is insignificant in the present data.

Both line features display pronounced radial velocity variations with a peak-to-peak amplitude of $500 \mathrm{~km} \mathrm{~s}^{-1}(\mathrm{H} \alpha)$ and $560 \mathrm{~km} \mathrm{~s}^{-1}(\mathrm{NaI})$, respectively. The sequence of five spectra obtained on March 10 rules out any period below $3 \mathrm{~h}$. A LombScargle periodogram of the radial velocities gives almost equal power at 5.28, 6.30, and 7.32 cycles per day (corresponding to periods of $270 \mathrm{~min}, 230 \mathrm{~min}$, and $200 \mathrm{~min}$, respectively). Without loss of generality we assume the shortest period to derive a spectroscopic ephemeris and to assign binary phases to individual spectra in this paper. The ephemeris of the blue-to-red zero crossing of the $\mathrm{NaI}$ lines thus derived is

$\mathrm{HJD}=2454537.2569(1)+E \times 0.1366(1)$ 


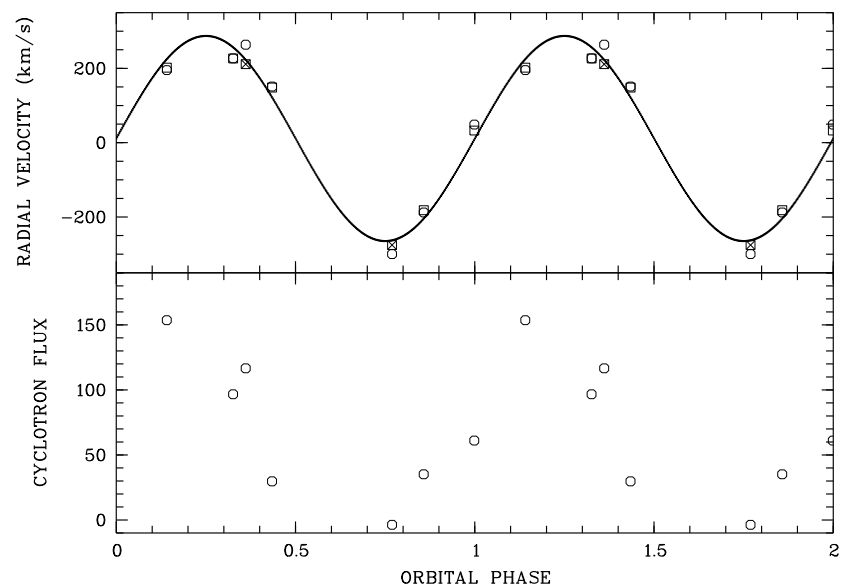

Fig. 3. Top panel: radial velocities of $\mathrm{H} \alpha$ emission (squares) and $\mathrm{Na}$ absorption lines (circles). The two data points from the first night are crossed. The continuous line is a sine fit to the Na radial velocities. Bottom panel: integrated cyclotron flux of the 4940 A spectral hump as a function of orbital phase. Flux units are $10^{-15} \mathrm{erg} \mathrm{cm}^{-2} \mathrm{~s}^{-1}$. The centroid of the bright orbital hump at phase 0.15 indicates the likely azimuth of the accretion spot.

The quoted $1 \sigma$ uncertainties were derived from a sine fit to the $\mathrm{NaI}$ radial velocities. The radial velocity curves of $\mathrm{H} \alpha$ and $\mathrm{NaI}$ lines are shown in Fig. 3. Both species display a common sinelike radial velocity curve, suggesting that $\mathrm{H} \alpha$ is due to stellar activity and not due to accretion.

\subsection{Continuum variability - the cyclotron spectrum}

The contribution of the assumed M4 ZAMS secondary star was subtracted from the seven individual spectra. These were then summed and a smoothed continuum was defined by a polynomial fit to 10 points in wavelength regions away from the spectral hump at $4940 \AA$. Again, the same smooth continuum was subtracted from all individual spectra. The residuals are shown in the original time sequence running from bottom to top in Fig. 4. Phases according to Eq. (1) are indicated (see also Table 1 for epochs and phases of individual spectra). The only remaining significant feature in the spectra apart from $\mathrm{H} \alpha$ is the broad hump which we interpret as a cyclotron harmonic in emission. The blue feature centered on $4200 \AA$ in spectrum \#1 cannot be classified. It may be a cyclotron line from a second region, but the flux changes rather abruptly and not as smoothly as seen in other cyclotron lines. It may therefore well be an artefact of the observation/reduction. Identification of this feature being either physical or instrumental needs further phase-resolved spectroscopy.

Although we could identify just one hump in the spectra we think there is little doubt about the nature of the feature being of cyclotron origin. The interpretation rests on the similarity to other systems, its variability pattern (again similar to other objects of this class), and the success of our modeling (see below). Final confirmation needs the identification of at least one neighbouring cyclotron harmonic or the detection of a polarized signal from the observed hump at $4940 \AA$.

Assuming the cyclotron interpretation is correct, some basic parameters of the radiation source can be derived/constrained, in the first place the strength of the magnetic field, $B$. A proper determination would require us to measure the separation between two adjacent harmonics, but the isolated harmonic already provides some strong constraints.

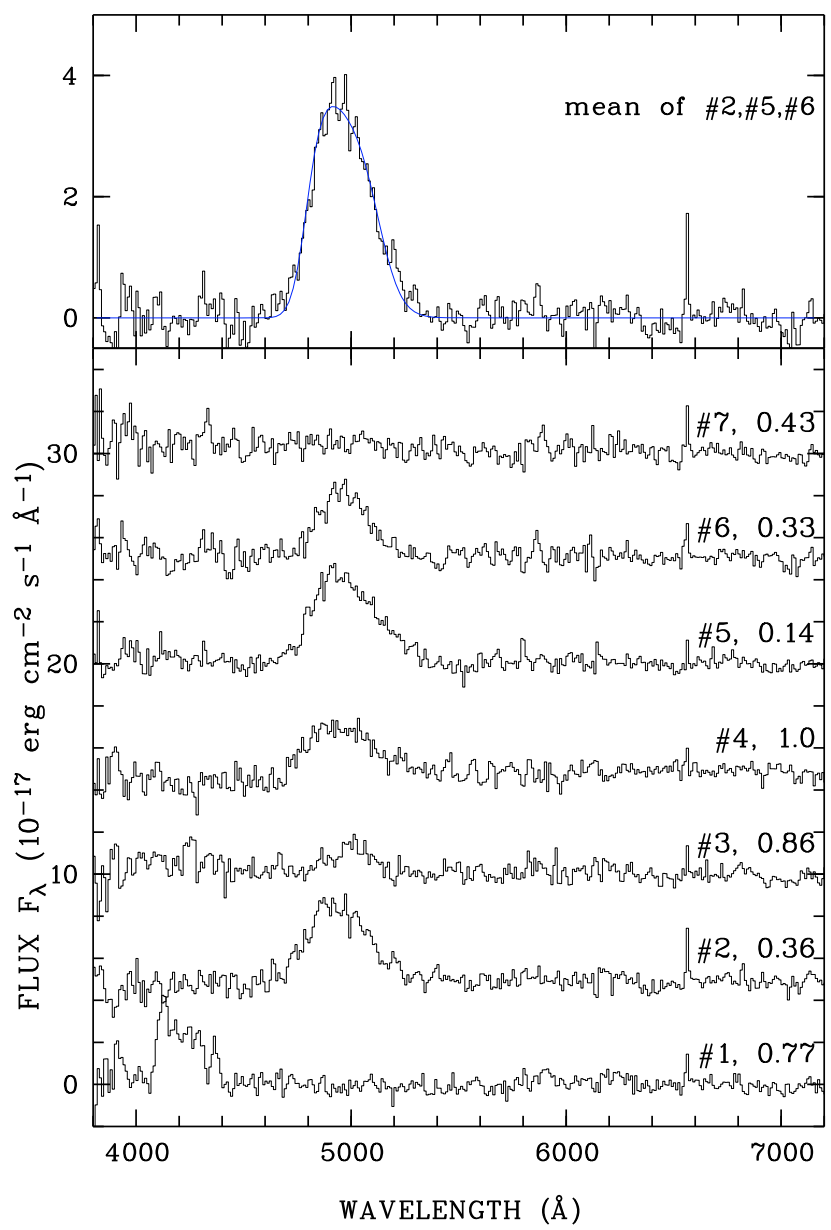

Fig. 4. Lower panel: cyclotron spectra of SDSS J120615.73+510047.0 generated by subtracting a ZAMS M4 template spectrum and a smooth blue continuum. The individual spectra were plotted with an offset of 5 flux units. Phases are indicated according to the ephemeris given in Eq. (1). Upper panel: mean of spectra with sequence numbers as indicated, compared with a cyclotron model emitted in a $1 \mathrm{keV}$ plasma at 108 MG.

Short vertical ticks in Fig. 2 indicate the expected positions of cyclotron harmonics $1-5$, if the observed one were the 1 st, 2nd, 3rd, or the 4th harmonic of the cyclotron fundamental in a field of $B=54.2,72.3,108.4$, or $216.8 \mathrm{MG}$, respectively. The fact that the one observed hump is isolated and well separated from its next (non-detected) neighbour excludes any field as low as $54 \mathrm{MG}$ (observed hump would be harmonic number $n=4$ ). A field strength of $72 \mathrm{MG}(n=3)$ seems unlikely too (but not completely excluded), since the 2 nd harmonic at $7500 \AA$ should be detectable. We remain with two alternatives, $B=108$ or $216 \mathrm{MG}$, and we are inclined to accept the former for the simple reason that the latter appears to be extraordinarily high.

The plasma temperature in the accretion region is low because the line does not show any significant wavelength shift, although the large photometric variability suggests that the viewing angle to the field is strongly variable. Furthermore, the line is rather narrow with $350 \AA(F W H M)$. Cyclotron spectra are determined by the magnetic field strength, the temperature, the viewing angle with respect to the field and the optical depth of the plasma at the given frequency (Schwope 1990). Assuming optically thin radiation and a moderate viewing angle of $60^{\circ}$, the plasma temperature can be crudely estimated. In Fig. 4 a model for the second harmonic in a $1 \mathrm{keV}$ plasma at $B=108 \mathrm{MG}$ 
is shown, which nicely represents the data (mean of the three brightest spectra).

The peak integrated flux of the cyclotron hump among the seven spectra is $F_{\text {cyc }}=1.5 \times 10^{-15} \mathrm{ergs} \mathrm{cm}^{-2} \mathrm{~s}^{-1}$ (see Fig. 3). If we assume a bolometric correction factor of 2 , the cyclotron luminosity is $L \simeq 2 F_{\text {cyc }} 2 \pi d^{2}=3 \times 10^{28}(d / 400 \mathrm{pc})^{2} \mathrm{ergs} \mathrm{s}^{-1}$. The implied mass accretion rate is estimated by equating the cyclotron luminosity to the accretion luminosity, $\dot{M} \simeq$ $10^{-14} M_{\odot} \mathrm{yr}^{-1}$. Although these numbers are uncertain, it is clear that SDSS J120615.73+510047.0 belongs to the class of lowluminosity, detached magnetic white-dwarf/main-sequence binaries found earlier in the HQS and the SDSS. The accretion rate is consistent with wind-accretion from the active secondary.

In Fig. 3 (lower panel) the optical cyclotron light curve is displayed as a function of the orbital phase. If one assumes spinorbit locking of the white dwarf, SDSS J120615.73+510047.0 has a cyclotron bright phase lasting for about 0.6 orbital cycles, centered on phase $\sim 0.1$. This phasing suggests that the accretion region is trailing the secondary by about 35 degrees in phase. This orientation is different from that which one typically finds in the polars, where the accreting pole is on the leading side (Cropper 1990). Spin-orbit synchronism is observed for all wellobserved objects of this class but needs to be proven for the new object by further photometry and spectroscopy.

\section{Discussion and conclusions}

We have identified SDSS J120615.73+510047.0 as a new member of the class of close, but still detached, binaries containing a late-type ZAMS dwarf and a cool magnetic white dwarf accreting at extremely low rate from a stellar wind of the secondary star.

What to call these objects? When fewer than a handful of such objects were known they were recognized as Low Accretion Rate Polars, or LARPs (Schwope et al. 2002b), an acronym now widely used. The implication of this naming convention was that these objects are ordinary polars that have entered an extended low state. This is true for e.g. EQ Cet (aka RBS206), which was considered a LARP because of its peculiar cyclotron spectrum (Schwope et al. 1999). However, it became later clear (Schmidt et al. 2005; Schwarz et al. 2001; Vogel et al. 2007) that the secondaries of most LARPs seem to be Roche-lobe underfilling and hence accretion via Roche-lobe overflow, the defining criterion of a cataclysmic variable, cannot occur and has not occurred in the past. Hence, they are precataclysmic binaries or more precisely, pre-polars, henceforth PREPs. Objects such as EQ Cet showing intermittent high accretion states (Schwope et al. 2002a) are consequently not members of this class.

We now discuss the system parameters of the ninth member and some of the class parameters of the PREPs. Based on the Sloan data alone, we could estimate the orbital period, the magnetic field strength of the white dwarf, the spectral type of the secondary, and the distance to the system. GALEX and 2MASS data were helpful to refine the stellar parameters.

The magnetic field of the white dwarf was identified via a remarkable spectral hump at $4940 \AA$ identified as the likely 2nd cyclotron harmonic in a low-temperature plasma with $B=$ 108 MG. Interestingly, none of the now nine members of this class displayed photospheric magnetism via Zeeman-split absorption lines, a likely combined effect of their faintness, their coolness and the expected large Zeeman spread in fields as high as 60-100 MG. If confirmed, the field of $108 \mathrm{MG}$ (or even

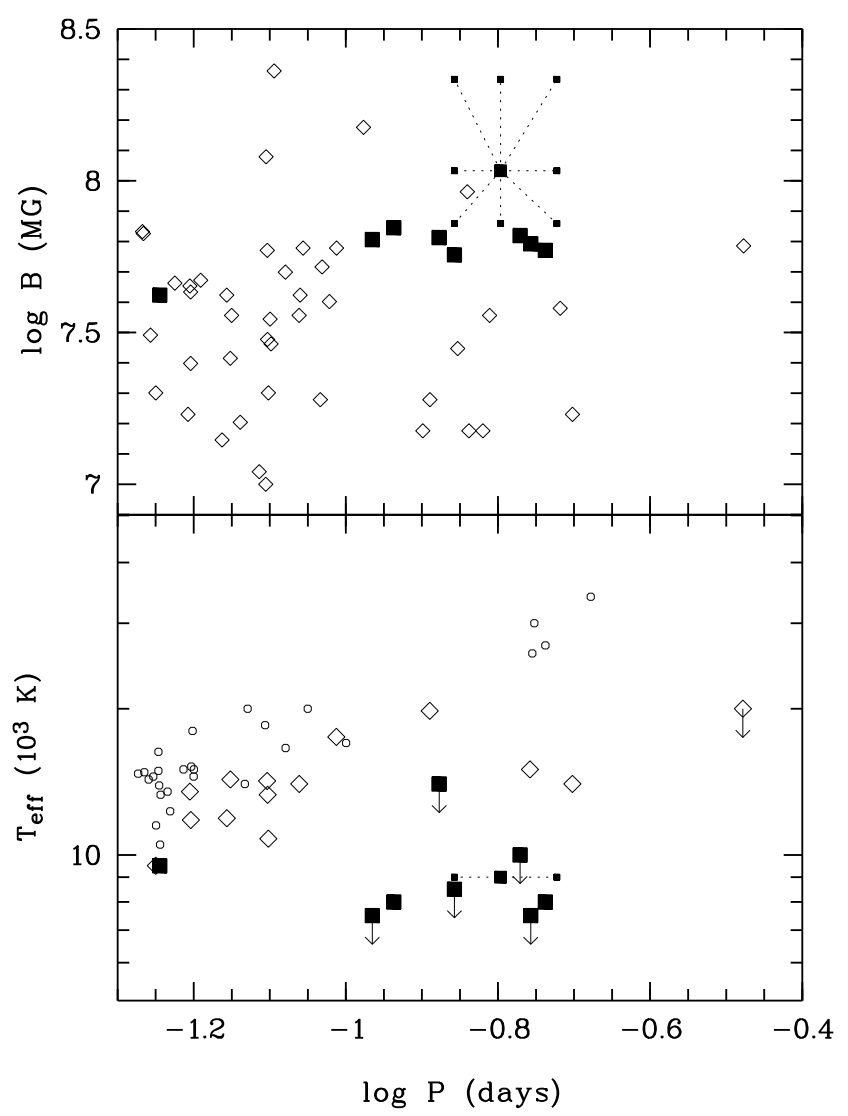

Fig. 5. Top: magnetic field versus orbital period of accreting close binaries containg a magnetic white dwarf and a main-sequence secondary. Open symbols denote binaries accreting via Roche-lobe overflow, the Polars. Filled symbols denote PREPs, filled symbols connected by dotted lines indicate possible locations of SDSS J120615.73+510047.0. Bottom: effective temperature of accreting magnetic and non-magnetic white dwarfs. Open circles indicate nonmagnetic white dwarfs, otherwise symbols are as above.

216 MG) in SDSS J120615.73+510047.0 extends the parameter range of PREPs to higher magnetic fields.

The white dwarf in SDSS J120615.73+510047.0 is cool, $T_{\text {eff }}<10000 \mathrm{~K}$, a common property of the class.

Polars and PREPs are thought to contain the same types of stars, magnetic white dwarfs and late-type ZAMS secondaries. While polars are accreting via Roche-lobe overflow with an estimated duty cycle of about $\sim 50 \%$ (Hessman et al. 2000), the latter are thought to accrete from the stellar wind of the secondary via a magnetic siphon (Schwope et al. 2002b; Webbink \& Wickramasinghe 2005; Schmidt et al. 2005; Vogel et al. 2007).

A comparison of the magnetic fields, the white-dwarf effective temperatures and the orbital periods between the polars and the PREPs is instructive and shown in Fig. 5. Values of the magnetic fields are based on our own compilation whereas the white-dwarf effective temperatures of polars and non-magnetic CVs are from the recent compilation by Townsley \& Gänsicke (2009). An upper limit temperature of $20000 \mathrm{~K}$ was added for V1309 Ori (Staude et al. 2001). Effective temperatures for PREPs are from Schmidt et al. (2005, 2007); Vogel et al. (2007) and this work.

Araujo-Betancor et al. (2005) and Townsley \& Gänsicke (2009) have discussed the distribution of $T_{\text {eff }}$ as a function of orbital period for non-magnetic and magnetic CVs (polars). They conclude that the temperature contrast between magnetic and 
non-magnetic white dwarfs that exists at any given period must arise from a difference in the time-averaged mass accretion rate, $\langle\dot{M}\rangle$. This is consistent with the suggestion that polars have lower angular momentum loss rates due to a reduced efficieny of magnetic braking (Wickramasinghe \& Wu 1994).

Figure 5 shows that the two classes containing a magnetic white dwarf, polars and PREPs, also separate rather well in temperature and magnetic field. All PREPs but one, SDSS J103100.6+202832.2 (Schmidt et al. 2007), have high magnetic fields and long orbital periods; most of them are found above the cataclysmic variable period gap. For MCV standards the objects thus appear mildly young. On the other hand, all have low white-dwarf effective temperatures which one might be tempted to regard as an age indicator.

Townsley \& Gänsicke (2009) have shown that compressional heating in polars is less efficient compared to non-magnetic CVs but still important. The separation between polars and PREPs in the $\left(T_{\text {eff }}, P\right)$ plane thus confirms that they are distinct classes, the WDs in polars being systematically hotter due to compressional heating. There is one interesting exception, SDSS J103100.6+202832.2, at the same temperature and orbital period as EF Eri, a well-studied polar with a cool white dwarf (Schwope et al. 2007). The striking resemblance between EF Eri and SDSS J103100.6+202832.2 was discussed by Schmidt et al. (2007) in their discovery paper. As a consequence of accretion heating, $T_{\text {eff }}$ will not serve as an age indicator for polars, but likely for PREPs due to their very low accretion rates.

Figure 5 shows that magnetic fields in PREPs cluster around 60-65 MG, with one clear exception, SDSS J103100.6+202832.2 at $42 \mathrm{MG}$, and another likely exception, SDSS J120615.73+510047.0 at 108 MG. While it is tempting to speculate about an evolutionary link between high magnetic fields on the one hand and long orbital periods/cool white dwarfs on the other hand, care must be taken about selection effects. The tenous, low-temperature plasmas in PREPs have radiative power only in the first few cyclotron harmonics. The cyclotron fundamental is likely optically thick (although not observed yet), and the fourth harmonic in several of the known cases is optically thin and rather difficult to detect. A straightforward detection by spectroscopic means as done in the past is more or less easily feasable, if the second or third harmonic lies between $4500 \AA$ and $\sim 8000 \AA$, corresponding to $B=45 \ldots 120 \mathrm{MG}$. The object SDSS J103100.6+202832.2 is slightly below this range. Its fortunate discovery was possible due to its rather high power in harmonics higher than the third while SDSS J120615.73+510047.0 is possibly close to the upper limit.

Low-field PREPs, i.e. those with cyclotron spectra in the infrared, could be recognized, by an infrared excess or if Zeeman-split photospheric lines from the white dwarfs could be identified. But even the known PREPs do not show any easily identifiable Zeeman feature in their flux spectra ${ }^{1}$ contrary to the white dwarfs in low-state polars which are hotter on average. This difference makes the identification of low-field PREPs practically very difficult. Low-field PREPs could be hidden among the WDMS-systems with apparent DC white dwarfs and would require either spectro-polarimetry to be identified or flux spectra with higher signal to noise ratios.

Whether the distribution of field strength between polars and PREPs is truly different or not remains unanswered for the time

\footnotetext{
1 Which is due to their low temperatures, hence their faintness and the small equivalent widths of the lines.
}

being, but their period distributions are very different and less affected by observational selection effects.

One explanation for the discrepant period distributions originally formulated by Webbink \& Wickramasinghe (2005) and later by Schmidt et al. (2005) assumes early synchronization of the white dwarfs in PREPs due to their (high?) magnetic field. Early synchronization was supposed to slow down binary evolution by an effective reduction or even complete cessation of magnetic braking above the orbital period gap. As a result, comparatively cool white dwarfs are observed even at long orbital periods. This scenario involves different angular momentum loss (AML) rates for polars and PREPs.

An alternative is to assume the same AML for both classes, the separation in $P$ then implies that PREPs are young compared to polars. Observationally both scenarios are difficult to discern, since $T_{\text {eff }}$ can not be used as age indicator.

Are the PREPs the missing detached magnetic white dwarf/main sequence binaries?

Liebert et al. (2005) noted the absence of non-accreting magnetic white dwarfs (MWDs) among the more than 1200 WDMS binaries compiled by Silvestri et al. (2007) and various other samples (Marsh 2000; Ritter \& Kolb 2003; Schreiber \& Gänsicke 2003; Morales-Rueda et al. 2005; Shimansky et al. 2006). MWDs are clearly underrepresented in current WDMS samples compared to the $\sim 10 \%$ fraction in the field (Liebert et al. 2003). As a first possible solution to this fact, Liebert et al. (2005) suggested that, analogous to single magnetic white dwarfs, the magnetic white dwarfs in WDMS binaries are more massive, and therefore smaller and less luminous compared to those in non-magnetic WDMS binaries which could result in an observational bias against their detection. However, Silvestri et al. (2007) convincingly demonstrate that such systems would be easily identified, if they were present in the SDSS data base.

Stimulated by the recent observational studies, Tout et al. (2008) propose a rather radical shift in paradigm for the formation of highly magnetic white dwarfs, suggesting that the formation of high-field white dwarfs in general is tightly related to the evolution through a common envelope phase. In that scenario, single high-field magnetic white dwarfs are the results of mergers during the common envelope, explaining the higher average mass of magnetic white dwarfs compared to non-magnetic white dwarfs. Those systems that avoid merging leave the common envelope as pre-polars with such short orbital periods that their white dwarfs can capture some of the wind of the companion star (in other words, they leave the CE as PREPs).

However, the cold temperatures of the white dwarfs in PREPs provide us with strong lower limits on their post common envelope lifetimes of the order of several hundred Myrs. Unfortunately, as the stellar masses of the PREPs remain unknown, we can neither determine the detailed cooling ages nor can we reconstruct their detailed post-CE evolution and, hence, cannot answer the question of whether they already could have been wind-accreting systems shortly after the common envelope phase. However, the fact that all PREPs contain cold white dwarfs, i.e. are old post common envelope binaries, implies that the appealing idea presented by Tout et al. (2008) does not solve the missing MWD problem but requires us to rephrase it: If PREPs are indeed the progenitors of polars, where are the progenitors of PREPS, i.e. WDMS systems containing hot magnetic white dwarfs?

Acknowledgements. We thank our referee, G.D. Schmidt, for valuable comments and suggestions which helped to improve the manuscript. We also thank Justus Vogel for comments on an earlier version of the manuscript. A.N.G.M. 
was supported by the Deutsches Zentrum für Luft- und Raumfahrt (DLR) GmbH under contract No. 50OR0404.

Funding for the SDSS and SDSS-II has been provided by the Alfred P. Sloan Foundation, the Participating Institutions, the National Science Foundation, the U.S. Department of Energy, the National Aeronautics and Space Administration, the Japanese Monbukagakusho, the Max Planck Society, and the Higher Education Funding Council for England. The SDSS Web Site is http://www . sdss.org/.

The SDSS is managed by the Astrophysical Research Consortium for the Participating Institutions. The Participating Institutions are the American Museum of Natural History, Astrophysical Institute Potsdam, University of Basel, University of Cambridge, Case Western Reserve University, University of Chicago, Drexel University, Fermilab, the Institute for Advanced Study, the Japan Participation Group, Johns Hopkins University, the Joint Institute for Nuclear Astrophysics, the Kavli Institute for Particle Astrophysics and Cosmology, the Korean Scientist Group, the Chinese Academy of Sciences (LAMOST), Los Alamos National Laboratory, the Max-Planck-Institute for Astronomy (MPIA), the Max-Planck-Institute for Astrophysics (MPA), New Mexico State University, Ohio State University, University of Pittsburgh, University of Portsmouth, Princeton University, the United States Naval Observatory, and the University of Washington.

\section{References}

Abazajian, K., Adelman-McCarthy, J., Agüeros, M., et al. 2009, ApJS, 182, 543 Araujo-Betancor, S., Gänsicke, B. T., Long, K. S., et al. 2005, ApJ, 622, 589

Cropper, M. 1990, Space Sci. Rev., 54, 195

Fukugita, M., Ichikawa, T., Gunn, J. E., et al. 1996, AJ, 111, 1748

Gunn, J. E., Carr, M., Rockosi, C., et al. 1998, AJ, 116, 3040

Gunn, J. E., Siegmund, W. A., Mannery, E. J., et al. 2006, AJ, 131, 2332

Hessman, F. V., Gänsicke, B. T., \& Mattei, J. A. 2000, A\&A, 361, 952

Hogg, D. W., Finkbeiner, D. P., Schlegel, D. J., \& Gunn, J. E. 2001, AJ, 122, 2129

Ivezić, Ž., Lupton, R. H., Schlegel, D., et al. 2004, Astron. Nachr., 325, 583

Leggett, S. K., Allard, F., Dahn, C., et al. 2000, ApJ, 535, 965

Liebert, J., Bergeron, P., \& Holberg, J. B. 2003, AJ, 125, 348

Liebert, J., Wickramsinghe, D. T., Schmidt, G. D., et al. 2005, AJ, 129, 2376

Marsh, T. R. 2000, New Astron. Rev., 44, 119

Morales-Rueda, L., Marsh, T. R., Maxted, P. F. L., et al. 2005, MNRAS, 359, 648
Nebot Gómez-Morán, A., Schwope, A. D., Schreiber, M. R., et al. 2009, A\&A, 495, 561

Pier, J. R., Munn, J. A., Hindsley, R. B., et al. 2003, AJ, 125, 1559

Rebassa-Mansergas, A., Gänsicke, B. T., Rodríguez-Gil, P., Schreiber, M. R., \& Koester, D. 2007, MNRAS, 382, 1377

Reimers, D., \& Hagen, H.-J. 2000, A\&A, 358, L45

Reimers, D., Hagen, H.-J., \& Hopp, U. 1999, A\&A, 343, 157

Ritter, H., \& Kolb, U. 2003, A\&A, 404, 301

Schmidt, G. D., Szkody, P., Vanlandingham, K. M., et al. 2005, ApJ, 630, 1037

Schmidt, G. D., Szkody, P., Henden, A., et al. 2007, ApJ, 654, 521

Schreiber, M., Nebot Gomez-Moran, A., Schwope, A., \& Gaensicke, B. 2009, A\&A, in prep.

Schreiber, M. R., \& Gänsicke, B. T. 2003, A\&A, 406, 305

Schreiber, M. R., Nebot Gomez-Moran, A., \& Schwope, A. D. 2007, in 15th European Workshop on White Dwarfs, ed. R. Napiwotzki, \& M. R. Burleigh, ASP Conf. Ser., 372, 459

Schwarz, R., Schwope, A. D., \& Staude, A. 2001, A\&A, 374, 189

Schwope, A. D. 1990, in Rev. Mod. Astron. 3, ed. G. Klare, 44

Schwope, A. D., Schwarz, R., \& Greiner, J. 1999, A\&A, 348, 861

Schwope, A. D., Brunner, H., Buckley, D., et al. 2002a, A\&A, 396, 895

Schwope, A. D., Brunner, H., Hambaryan, V., \& Schwarz, R. 2002b, in The Physics of Cataclysmic Variables and Related Objects, ed. B. T. Gänsicke, K. Beuermann, \& K. Reinsch, ASP Conf. Ser., 261, 102

Schwope, A. D., Staude, A., Koester, D., \& Vogel, J. 2007, A\&A, 469, 1027

Shimansky, V., Sakhibullin, N. A., Bikmaev, I., et al. 2006, A\&A, 456, 1069

Silvestri, N. M., Lemagie, M. P., Hawley, S. L., et al. 2007, AJ, 134, 741

Smith, J. A., Tucker, D. L., Kent, S., et al. 2002, AJ, 123, 2121

Staude, A., Schwope, A. D., \& Schwarz, R. 2001, A\&A, 374, 588

Stoughton, C., Lupton, R. H., Bernardi, M., etal. 2002, AJ, 123, 485

Tout, C. A., Wickramasinghe, D. T., Liebert, J., Ferrario, L., \& Pringle, J. E. 2008, MNRAS, 387, 897

Townsley, D. M., \& Gänsicke, B. T. 2009, ApJ, 693, 1007

Tucker, D. L., Kent, S., Richmond, M. W., et al. 2006, Astron. Nachr., 327, 821

Vogel, J., Schwope, A. D., \& Gänsicke, B. T. 2007, A\&A, 464, 647

Webbink, R. F., \& Wickramasinghe, D. T. 2005, in The Astrophysics of Cataclysmic Variables and Related Objects, ed. J.-M. Hameury, \& J.-P. Lasota, ASP Conf. Ser. 330, 137

Wickramasinghe, D. T., \& Wu, K. 1994, MNRAS, 266, L1

Yanny, B., Rockosi, C., Newberg, H. J., et al. 2009, AJ, 137, 4377

York, D. G., Adelman, J., Anderson, Jr., J. E., et al. 2000, AJ, 120, 1579 